\title{
A Role for Blind DN2 Clock Neurons in Temperature Entrainment of the Drosophila Larval Brain
}

\author{
Marie Picot, ${ }^{\star}$ André Klarsfeld, ${ }^{\star}$ Elisabeth Chélot, Sébastien Malpel, and François Rouyer \\ Institut de Neurobiologie Alfred Fessard, Centre National de la Recherche Scientifique (Unité Propre de Recherche 2216), 91198 Gif-sur-Yvette, France
}

Circadian clocks synchronize to the solar day by sensing the diurnal changes in light and temperature. In adult Drosophila, the brain clock that controls rest-activity rhythms relies on neurons showing Period oscillations. Nine of these neurons are present in each larval brain hemisphere. They can receive light inputs through Cryptochrome (CRY) and the visual system, but temperature input pathways are unknown. Here, we investigate how the larval clock network responds to light and temperature. We focused on the CRY-negative dorsal neurons (DN2s), in which light- dark (LD) cycles set molecular oscillations almost in antiphase to all other clock neurons. We first showed that the phasing of the DN2s in LD depends on the pigment-dispersing factor (PDF) neuropeptide in four lateral neurons (LNs), and on the PDF receptor in the DN2s. In the absence of PDF signaling, these cells appear blind, but still synchronize to temperature cycles. Period oscillations in the DN2s were stronger in thermocycles than in LD, but with a very similar phase. Conversely, the oscillations of LNs were weaker in thermocycles than in LD, and were phase-shifted in synchrony with the DN2s, whereas the phase of the three other clock neurons was advanced by a few hours. In the absence of any other functional clock neurons, the PDF-positive LNs were entrained by LD cycles but not by temperature cycles. Our results show that the larval clock neurons respond very differently to light and temperature, and strongly suggest that the CRY-negative DN2s play a prominent role in the temperature entrainment of the network.

\section{Introduction}

Circadian clocks involve self-sustained molecular oscillations of $\sim 24$ h periodicity (Dunlap et al., 2004; Bell-Pedersen et al., 2005), synchronized to the day-night cycles by light and temperature (Rensing and Ruoff, 2002; Roenneberg et al., 2003). In Drosophila, circadian entrainment has been mainly investigated in the adult clock that controls activity rhythms (Hall, 2003). It contains $\sim 80$ clock neurons per hemisphere, which are divided into several lateral and dorsal groups (Chang, 2006; Shafer et al., 2006). In light-dark (LD) conditions, the lateral neurons (LNs) that express the pigment-dispersing factor (PDF) drive morning activity, whereas PDF-negative neurons drive evening activity (Grima et al., 2004; Stoleru et al., 2004). In LD, $p d f^{01}$ mutants display an

\footnotetext{
Received Jan. 21, 2008; revised March 13, 2009; accepted March 16, 2009
}

This work was supported by Action Concertée Incitative“Biologie du Développement et Physiologie Intégrative" from Ministère de I'Enseignement Supérieur et de la Recherche (MESR), Agence Nationale de la Recherche "Neurosciences, Neurologie et Psychiatrie," and European Union Sixth Framework Project "EUCLOCK" (F.R.). M.P. was supported successively by MESR and Fondation pour la Recherche Médicale, and F.R. was supported by Institut National de la Santé et de la Recherche Médicale. We thank B. Richier for his expertise with the imaging system, M Boudinot for the Faas software, G. Levesque for helping with the behavioral setup, and Carine Vias for her help with dissections. We thank the Imagif facility for confocal microscopy. We are grateful to J. Champagnat for his strong support. We also thank M. Rosbash for providing the UAS-cry line, J. Kim for han mutants and UAS-han flies, and R. Stanewsky for anti-PER serum.

*M.P. and A.K. contributed equally to this work.

Correspondence should be addressed to François Rouyer, Institut de Neurobiologie Alfred Fessard, Centre $\mathrm{Na}$ tional de la Recherche Scientifique (Unité Propre de Recherche 2216), Avenue de la Terrasse, 91198 Gif-sur-Yvette Cedex, France. E-mail: rouyer@inaf.cnrs-gif.fr.

S. Malpel's present address: Unité Mixte de Recherche 5548, Centre National de la Recherche Scientifique and Université de Bourgogne, 21000 Dijon, France.

M. Picot's present address: Unité Mixte de Recherche 7224, Centre National de la Recherche Scientifique and Université Pierre et Marie Curie, 75005 Paris, France.

DOI:10.1523/JNEUROSCI.0279-08.2009

Copyright $\odot 2009$ Society for Neuroscience $\quad 0270-6474 / 09 / 298312-09 \$ 15.00 / 0$ advanced evening activity peak and lack the morning activity peak (Renn et al., 1999; Grima et al., 2004). No molecular phenotype has been associated with the behavioral defects of $p d f^{01}$ flies in LD.

Light entrains the adult circadian network through both rhodopsin-containing photoreceptors and the blue-lightsensitive protein Cryptochrome (CRY), acting cell autonomously in most clock neurons (Emery et al., 2000; HelfrichFörster et al., 2001, 2002; Ivanchenko et al., 2001; Rieger et al., 2003; Klarsfeld et al., 2004; Veleri et al., 2007). Temperature cycles entrain activity rhythms in constant darkness (Wheeler et al., 1993) and even in constant light (Matsumoto et al., 1998), which abolishes activity rhythms in constant temperature (Konopka et al., 1989). Interestingly, temperature entrainment is more efficient on at least some PDF-negative neurons, whereas the PDFexpressing neurons are more responsive to light (Yoshii et al., 2005; Busza et al., 2007; Miyasako et al., 2007). Genes involved in temperature resetting have been described, but its mechanisms are still mostly hypothetical (Sidote et al., 1998; Glaser and Stanewsky, 2005; Boothroyd et al., 2007; Kaushik et al., 2007; Yoshii et al., 2007).

The brain clock network is much simpler in the larva (Kaneko et al., 1997): five LNs, four of which express PDF, and two pairs of dorsal neurons (DNs) (DN1s and DN2s). Only the PDF-positive LNs and the DN1s express CRY (Klarsfeld et al., 2004). The larval visual nerve projects onto the PDF-expressing LNs (Kaneko et al., 1997; Malpel et al., 2002). CRY and the rhodopsin-containing visual organ provide the only light inputs into the clock (Kaneko et al., 2000; Malpel et al., 2004). The DN2s lack any obvious visual afferences (Kaneko and Hall, 2000), but lie close to axonal terminals of the LNs. Intriguingly, they display Period (PER) oscilla- 
A
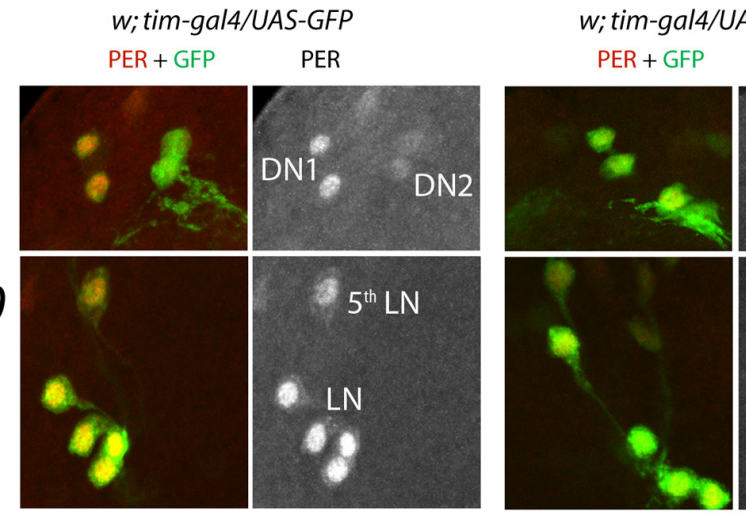

S-GFP; $p d f$

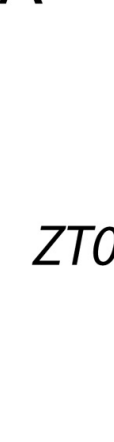

PER
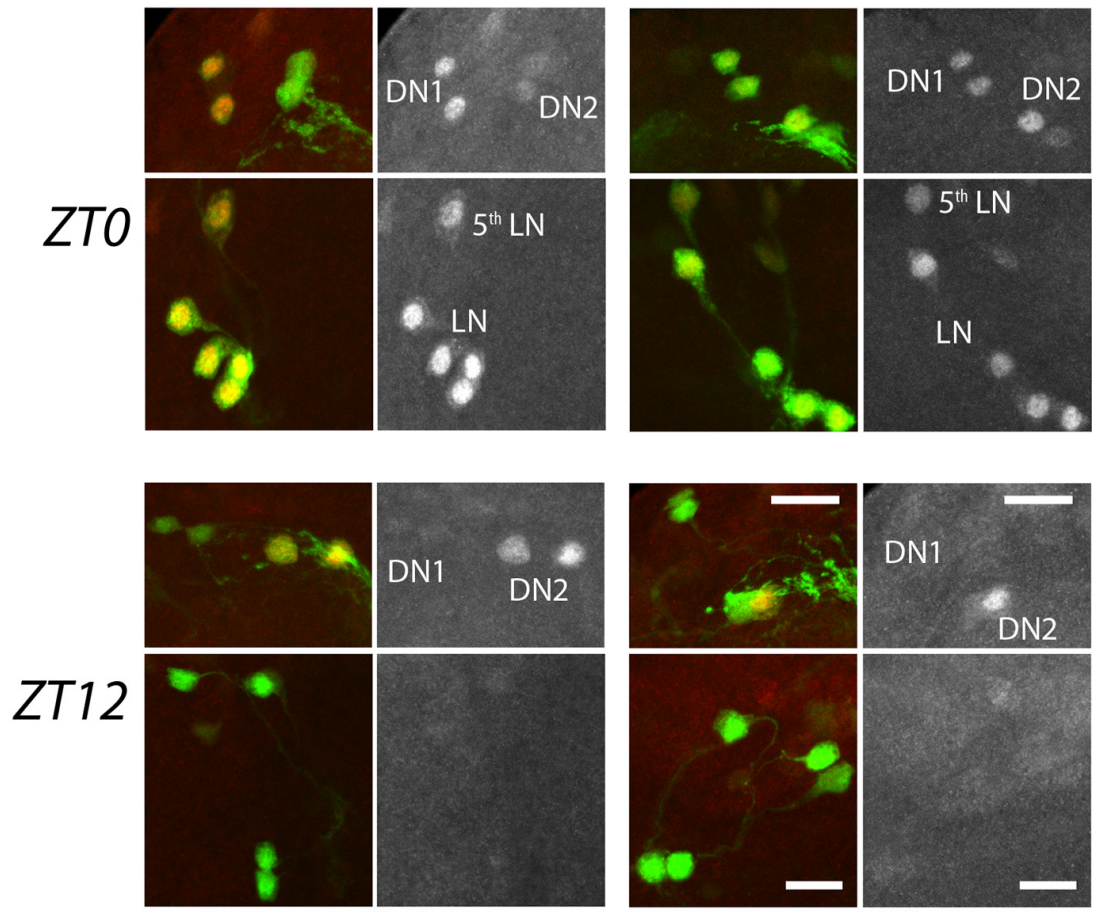

B

w; tim-gal4/UAS-GFP
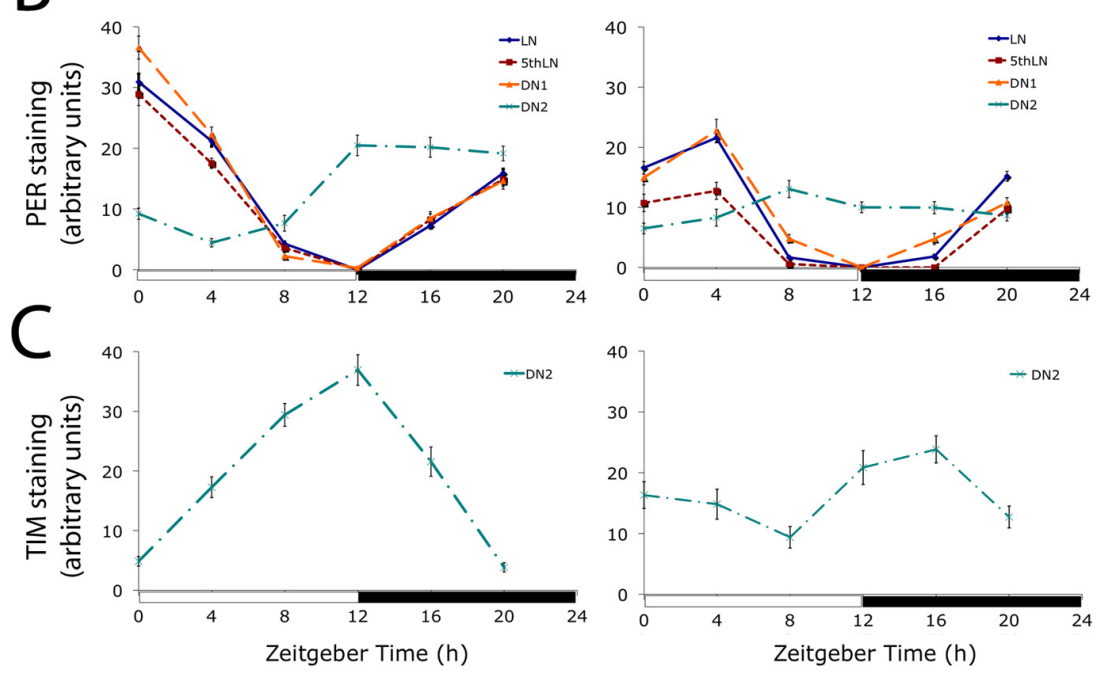

Figure 1. PER and TIM labeling in wild-type (left panels) and $p d f^{01}$ (right panels) larval brains in LD. Larvae were entrained in $L D$ conditions for $5 \mathrm{~d}\left(25^{\circ} \mathrm{C}\right)$ before dissection of $15-20$ brain hemispheres for each time point. The GFP reporter expressed under tim-gal 4 driver control was used to label each neuronal group. $A$, PER immunoreactivity in wild-type and $p d f^{01}$ mutant brains at ZTO (light on) and ZT12 (light off). Representative regions of the LNs and DNs are shown as projections of confocal stacks. In the wild type at ZT0, five LNs and two DN1s are strongly labeled, whereas the DN2s are only weakly labeled. At ZT12, no more PER labeling is visible in LNs and DN1s, whereas DN2s show stronger labeling. PER labeling in the LNs and the DN1s is unchanged in $p d f^{17}$ mutants compared with wild type. In the DN2s of the mutant, however, no clear difference in labeling was observed between ZT0 and ZT12. Note also the heterogeneity in PER localization at both time points in the mutant. $\boldsymbol{B}$, Time course of PER oscillations in different groups of larval clock neurons. Brains were dissected every $4 \mathrm{~h}$ during an LD cycle. The white and black boxes on $x$-axes indicate light and dark periods, respectively. PER labeling was quantified in the four PDF-positive LNs, the fifth LN, and the DN1s and DN2s, as indicated. Error bars represent the SEM for each neuronal group. Very similar results were obtained in two other experiments for each genotype. Although ANOVA found significant effects of time on PER levels in the DN2s of the $p d f^{0}$ mutant, their oscillations were clearly blunted. For instance, in the experiment shown here, post hoc Dunnett's test concluded that the ZT4 time point differed only from the ZT8 time point $(p<0.05)$ in the mutant, while differing from ZTO $(p<0.05)$, ZT12, ZT16, and ZT20 ( $p<0.001)$ in the wild type. C, Time course of TIM oscillations in the larval DN2s. This was obtained as in $\boldsymbol{B}$ in an experiment in which TIM and PER were both labeled. Again, ANOVA found significant effects of time on TIM levels in the DN2s of the $p d f^{0}$ mutant, but post hoc Dunnett's test concluded that the ZTO time point was not significantly different from any other time point $(p>0.05)$ in the mutant, while differing from all other time points $(p<0.001)$ except ZT20 in the wild type. tions almost in antiphase to all other clock neurons (Kaneko et al., 1997), and forced expression of CRY in these cells reverses their phase (Klarsfeld et al., 2004). This suggests that the DN2s perceive light only through the clock network. In contrast, thermocycles might entrain all clock neurons in a cell-autonomous way.

We first tested whether PDF and its receptor are involved in light setting the unique phase of the larval DN2s. We obtained direct evidence for a strong role of PDF, also suggesting that PDF acts on its receptor in the DN2s themselves. We then found that thermocycles phase the larval clock neurons very differently from light entrainment, bringing the PDF-positive LNs in phase with the DN2s. Entrainment of larvae in which PER expression was restricted to specific subsets of clock neurons indicated that the LN clock is temperature blind in the absence of PER oscillations in other clock neurons. Our results thus strongly suggest that the larval DN2s play a specific role in temperature entrainment.

\section{Materials and Methods}

Strains. Drosophila cultures were usually maintained on a $12 \mathrm{~h}$ LD cycle on standard corn meal-yeast-agar medium at $25^{\circ} \mathrm{C}$ and $50 \%$ relative humidity. Depending on the temperature conditions, the developmental time varies between 5 and $7 \mathrm{~d}$ to reach the wandering thirdinstar larval stage, at which the immunostaining experiments were done, and between 17 [hot/cold/cold (HCC)] and 20 (LDD) days to obtain the adults used for the behavioral experiments. Although we cannot exclude that developmental time could differently affect different groups of clock neurons, temperature differences during development, by themselves, do not seem to affect the relative phases of the clock neurons, as judged from dissecting brains from larvae raised at $25^{\circ} \mathrm{C}$ or $19^{\circ} \mathrm{C}$ in LD (S. Malpel, A. Klarsfeld, and F. Rouyer, unpublished results). Light conditions had no obvious effect on developmental time. Control strains were laboratory stocks of $w$ or $y w$ flies, which behaved similarly. han ${ }^{5304}$ and UAS-han (CG13758) (Hyun et al., 2005), pdf ${ }^{01}$ (Renn et al., 1999), UAS-cry (Emery et al., 2000), crygal4-39 (Klarsfeld et al., 2004), pdf-gal4 (Park et al., 2000), UAS-per16 (Blanchardon et al., 2001), cry-gal80 (Stoleru et al., 2004), UAS-hid and UAS-rpr (Zhou et al., 1997) lines have been previously described. Simultaneous $p d f$-gal4-driven hid and rpr expression eliminates the LNs under standard culture conditions (Malpel et al., 2002). We checked that the same held true in temperature cycles $\left(25-19^{\circ} \mathrm{C}\right)$. yw; tim-gal4 is expressed in all clock neurons of the larval brain and all clock neurons of the adult brain in addition to several other neuronal groups that do not express clock genes (Kaneko, 1998). The UAS-GFP and UAS-CD8-GFP re- 


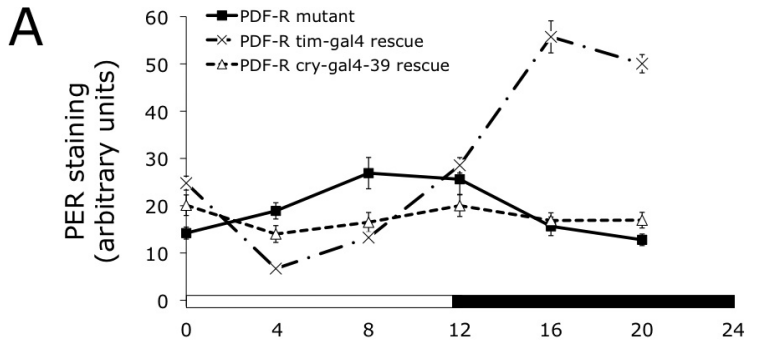

B

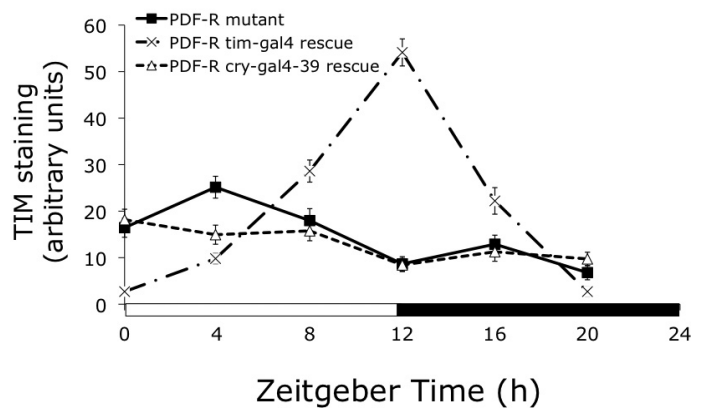

Figure 2. Time course of molecular oscillations in the DN2s of PDF-R mutant larvae, and their rescue. Fly entrainment and PER and TIM quantification were performed as described in Figure 1. Brains were dissected every $4 \mathrm{~h}$ during an LD cycle. The white and black boxes on $x$-axes indicate light and dark periods, respectively. Error bars represent the SEM at each time point. $\boldsymbol{A}$, $\boldsymbol{B}$, PER $(\boldsymbol{A})$ and TIM $(\boldsymbol{B})$ oscillations in the DN2s of PDF-R mutant larvae, and in mutant larvae with PDF receptor rescue in all clock cells (tim-gal4 driver) or only the four PDF-positive LNs and the DN1s (cry-gal4-39 driver). ANOVA concluded that cry-gal4-39-rescued PER and TIM oscillations were not significantly different $(p>0.15)$ from those in the PDF-R mutant.

porter lines carry their insertion on the second chromosome and were provided by the Bloomington Stock Center.

Culture and entrainment conditions. For light entrainment experiments, LD-grown flies were crossed and placed in LD conditions at $25^{\circ} \mathrm{C}$. At this temperature, the offspring reach third-instar wandering stage in $\sim 5 \mathrm{~d}$. Cultures were thus entrained in LD 12:12 for 5 or $6 \mathrm{~d}$ before larvae were dissected (for LD time points), or entrained in LD 12:12 for $4 \mathrm{~d}$ and transferred into constant darkness $24 \mathrm{~h}$ before first dissection time [circadian time 12 (CT12)]. For temperature entrainment experiments, after $1 \mathrm{~d}$ in $\mathrm{LD}$ at $25^{\circ} \mathrm{C}$ to initiate mating and egg laying, cultures were reared in $\mathrm{DD} 25^{\circ} \mathrm{C}(12 \mathrm{~h}) / 19^{\circ} \mathrm{C}(12 \mathrm{~h})$ thermocycles [hot/cold (HC)]. The warm interval has been defined as Zeitgeber time 0 (ZT0) to ZT12. Under these conditions, the offspring reach third-instar wandering stage in $\sim 7 \mathrm{~d}$. Cultures were thus entrained in HC for 7 or $8 \mathrm{~d}$ before larvae were dissected (for HC time points, ZT0 to ZT20), or transferred into constant conditions (darkness at $\left.19^{\circ} \mathrm{C}\right) 24 \mathrm{~h}$ before first dissection time (CT12). Such larvae had never seen light. The Drosophila brain clock does not need light to start, although it is then unsynchronized in different individuals (Sehgal et al., 1992; Malpel et al., 2004). When per expression was driven via the GAL4-UAS system in $25-19^{\circ} \mathrm{C}$ HC cycles, high levels of PER (often prominently cytoplasmic) were observed at both ZT0 and ZT12 (data not shown). GAL4 activity is known to increase with temperature. We reasoned that such variations, by altering per transcription rate from the UAS construct, might mask PER regulation by the clock. Additionally, expression at $25^{\circ} \mathrm{C}$ might be too strong, thus overwhelming PER regulatory mechanisms (Zeng et al., 1994; Blanchardon et al., 2001). Indeed, in LD, PER oscillations in the UAS-per16-based larval brain clocks were stronger at $20^{\circ} \mathrm{C}$ than at $25^{\circ} \mathrm{C}$ (data not shown). To avoid these two pitfalls, entrainment for per rescue experiments in $\mathrm{HC}$ was thus performed with $23-18^{\circ} \mathrm{C} \mathrm{HC}$ cycles, and brains were dissected during the second day in constant conditions $\left(\mathrm{DD}, 18^{\circ} \mathrm{C}\right)$.

Histology and imaging. All experiments were done on whole-mounted third-instar larval brains, which were dissected as described previously (Malpel et al., 2002). The rabbit anti-PER antiserum (Stanewsky et al., 1997) was not adsorbed against adult per $^{0}$ acetone powder and was used at 1:15,000 dilution. The rat anti-Timeless (TIM) antiserum (Grima et al., 2002) and the mouse anti-PDF antiserum (Developmental Studies Hybridoma Bank) were used at 1:10,000 and 1:50,000 dilutions, respectively. Secondary antibodies were Alexa 594-, FP546- (or FP568-), and FP647-conjugated goat antibodies rabbit IgG (Invitrogen). They were used at 1:10,000, 1:5000, and 1:5000 dilutions, respectively. In tim-gal4/ UAS-GFP larvae, the fifth PDF-negative LN was identified by a combination of several criteria: its tim-gal4-driven green fluorescent protein (GFP) labeling is almost always weaker that the labeling of the other four LNs, it is slightly larger and more oblong, and it is usually positioned apart (and rather dorsal) from the other four LNs (see Fig. $1 A$ ). Images were made from an epifluorescence microscope (Axioplan2 or Z1; Zeiss) with a cooled digital camera (SPOT2; Diagnostic Instruments; or Axiocam MR; Zeiss). Fluorescence intensity was quantified with the Adobe Photoshop or ImageJ software from digital images of 15-20 brain hemispheres per sample. We applied the formula: $I=100(S-B) / B$, which gives the fluorescence percentage above background (where $S$ is fluorescence intensity and $B$ is average intensity of the region adjacent to the positive cell). Absolute values of $I$ varied between experiments, especially when imaged on a different microscope (hence the higher values in the left panels of Fig. $3 A$ and in Fig. $4 A$, relative to the other panels of the same figures). Therefore, only relative variations (between time points, or between cell types) within one experiment can be taken into consideration. Confocal imaging at higher magnification was performed on a Leica SP2 microscope. Maximum intensity projections were obtained from stacks of 5-15 confocal sections. Statistical analyses were performed with Prism (GraphPad Software).

Behavioral analyses. For larval-only light entrainment (LDD), cultures were first kept in $\mathrm{LD}$ at $20^{\circ} \mathrm{C}$, and transferred to constant darkness before pupariation started. For larval-only temperature entrainment (HCC), cultures were first kept in $\mathrm{HC}$, as described above, and transferred to constant temperature $\left(19^{\circ} \mathrm{C}\right)$ before pupariation started. In both cases, eclosed adults were put into monitors under red safelight (Kodak GBX-2 filter, with cutoff at $610 \mathrm{~nm}$ ) (Malpel et al., 2004). For adult light entrainment (LD), cultures were kept in $\mathrm{LD}$ at $20^{\circ} \mathrm{C}$ all along. Locomotor activity assays were always done with young adults (1-5 d of age) in constant darkness at $20^{\circ} \mathrm{C}\left( \pm 0.5^{\circ} \mathrm{C}\right)$, using commercially available monitors (Trikinetics). Raw activity data in DD (5-6 d) were analyzed on a Macintosh computer with the FaasX software (Klarsfeld et al., 2003), which is adapted from the Brandeis Rhythm Package (Hamblen et al., 1986). Individual flies are considered rhythmic when power $>20$, width $>1.5$, and $18 \mathrm{~h}<$ period $<30 \mathrm{~h}$. Waveforms of individual rhythmic flies are activity profiles averaged over several circadian cycles (which are defined for each fly according to its own period, as computed by Faas). They allow determination of the peak phase (Pha) of activity for that fly, also expressed in circadian time. A mean waveform and a mean phase can then be computed for each fly genotype. Circular statistics are used to determine significance and $95 \%$ confidence limits of mean phases. Mean phases can also be determined graphically on the mean waveforms.

\section{Results}

\section{PDF mediates entrainment of the larval DN2s by light}

In LD conditions, PER levels oscillate approximately in antiphase with the DN1s and the LNs in wild-type larval DN2s (Kaneko et al., 1997). We compared wild-type and $p d f^{01}$ mutant brains in a detailed LD time course. In $p d f^{\rho 1}$ mutants, PER oscillations were severely dampened in the DN2s, with moderate protein levels (Fig. $1 A, B$; supplemental Fig. $1 A, B$, available at www.jneurosci. org as supplemental material). In contrast, the temporal pattern of PER expression in the DN1s, the PDF-expressing LNs, and the PDF-negative fifth LN was little affected in the mutant, with trough levels at ZT12. Similar results were obtained for TIM levels: a marked dampening of cycling in the DN2s (Fig. 1C) and little or no change in the other neurons (data not shown).

Lack of entrainment of the DN2s in the $p d f^{01}$ mutant was also evident in their temporal pattern of PER subcellular localization, which did not display any clear oscillations, in contrast with the wild-type pattern (supplemental Fig. $2 A, B$, available at www. 

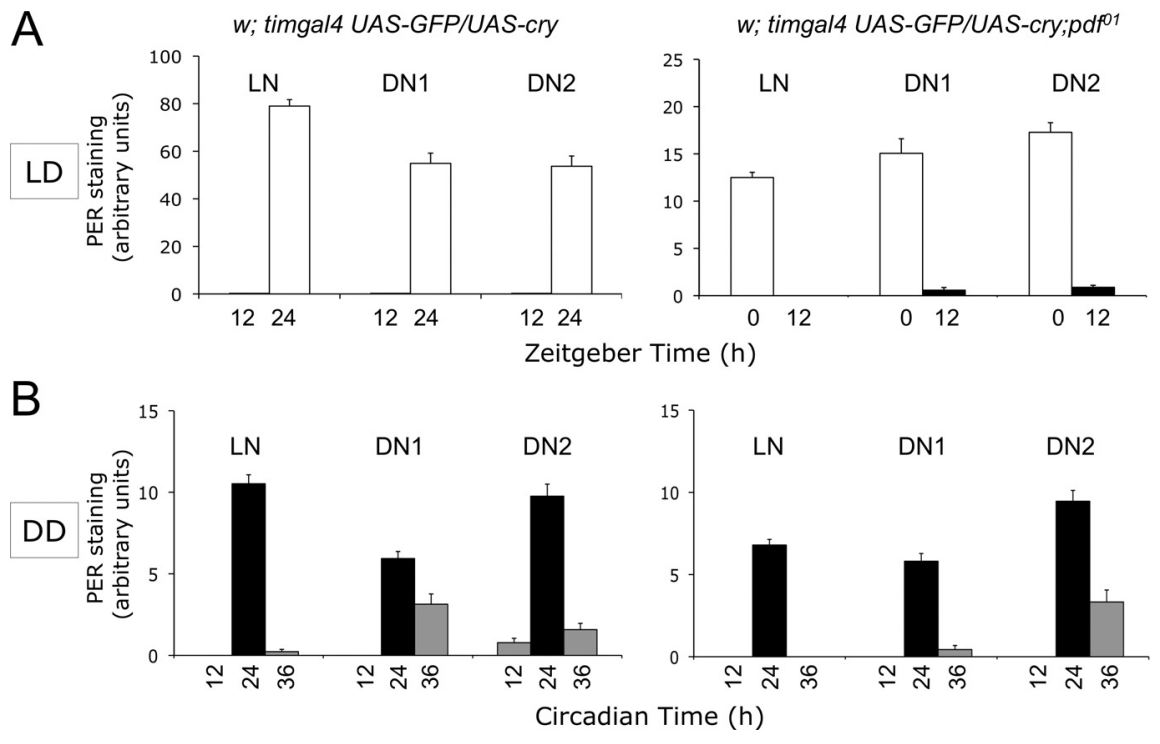

Figure 3. PER oscillations in clock neurons of larvae with CRY-expressing DN2s, in the presence or absence of PDF. Fly entrainment and PER quantification were performed as described in Figure 1, except that all five LNs were quantified together since there was no difference between the oscillations of the four PDF-expressing LNs and those of the fifth (PDF-negative) LN. Brains were dissected at predicted peak and trough times of PER expression in $\mathrm{LD}(\boldsymbol{A})$ or during the 2 first days of $D D(\boldsymbol{B})$, with CT0 corresponding to the end of the last LD cycle (when lights do not come back on). Error bars represent the SEM for each neuronal group.

tained in the absence of light input (Fig. $3 B)$. We conclude that forced CRY expression in the DN2s renders them independent from PDF signaling in LD and DD conditions (at least for the first $36 \mathrm{~h}$ of DD).

\section{Temperature entrainment brings the PDF-expressing LNs in phase with the DN2s}

The larval clock can be entrained by temperature, as assessed from the activity of completely blind adults that develop from such temperature-entrained larvae (Malpel et al., 2004). We thus asked how temperature entrainment affects the blind $\mathrm{DN} 2 \mathrm{~s}$ and the other larval clock neurons, in the absence of light. In temperature entrainment (HC, for hot/cold cycles), PER levels oscillated in all clock neurons (Fig. $4 A$; supplemental Fig. $1 C$, available at www.jneurosci.org as supplemental material). Interestingly, PER oscillations in the $\mathrm{DN} 2 \mathrm{~s}$ were more robust in $\mathrm{HC}$ than in LD cycles, whereas they were dampened in the other clock neurons, especially in the LNs

jneurosci.org as supplemental material). For instance, in the wild type, PER appeared exclusively nuclear in $>95 \%$ of DN2s at ZT16-ZT20 but in $<10 \%$ at ZT8-ZT12. In the $p d f^{01}$ mutant, PER appeared exclusively nuclear in $\sim 50 \%$ of the cells at all times, supporting lack of entrainment rather than lack of cycling.

\section{Light entrainment of the DN2s requires the PDF receptor}

We next tested the entrainment of the DN2s by light in larvae carrying $\mathrm{han}^{5304}$, a strong loss-of-function mutation of the gene encoding the PDF receptor (PDF-R) (Hyun et al., 2005). PER oscillations in the DN2s of the mutant larvae were strongly dampened (Fig. 2A), similarly to those measured in the $p d f^{01}$ mutant (Fig. $1 B$ ). When PDF-R expression was restored in all clock neurons of the han ${ }^{5304}$ mutant, including the DN2s, PER oscillations were fully restored. In contrast, when PDF-R expression was restored only in other cells (LNs and DN1s), PER oscillations in the DN2s were not restored (Fig. 2A). Similar results were obtained for TIM oscillations (Fig. $2 B$ ). These targeted rescue experiments strongly suggest that the PDF receptor is expressed in wild-type larval DN2s, in which it receives the entraining PDF signal from the LNs.

\section{Targeted expression of CRY in the larval DN2s makes them independent of PDF signaling}

CRY is not expressed in the larval DN2s, and forced CRY expression in those cells brings their PER oscillations in phase with the other clock neurons (Klarsfeld et al., 2004) (Fig. 3A). The same targeted expression experiment was performed in a $p d f^{01}$ background. As shown in Figure 3A, CRY-expressing larval DN2s entrain to LD cycles in the absence or presence of PDF signaling, with the same phase as the other clock neurons. Since wild-type larval DN2s keep their antiphase cycling when transferred from LD to DD (Kaneko et al., 1997, 2000), we asked whether CRYexpressing DN2s would revert to their normal antiphase cycling in DD. At least for the first $36 \mathrm{~h}$ in DD, PER oscillations maintained their phase in the presence or absence of PDF signaling, indicating that the CRY-induced phase change could be main- (compare Figs. $4 A, 1 B$ ). Similar results were obtained for TIM levels (data not shown).

The most striking difference between temperature and light entrainment was the phase of the various neuronal groups. Only the DN2s appeared similarly phased in both LD and HC conditions, with a trough of PER levels at ZT4 and peak at ZT12-ZT16. In the DN1s and the fifth LN, PER oscillations were somewhat advanced in $\mathrm{HC}$ relative to LD cycles (trough at ZT8 in HC vs ZT12 in LD). The phase of the four PDF-positive LNs was completely changed in HC cycles, with PER trough at ZT4 and peak at ZT16, now identical with the phase of the DN2s (Fig. $4 A$; supplemental Fig. $1 C$, available at www.jneurosci.org as supplemental material). Subcellular PER localization was also similar in the PDF-positive LNs and in the DN2s, becoming more nuclear as protein levels increased from their trough at ZT4 to their peak at ZT12-ZT16 (supplemental Fig. 2C, available at www.jneurosci. org as supplemental material). Thus, temperature cycles induce two distinct types of oscillations in the larval clock neurons, with the PDF-positive LNs and the DN2s cycling together with at least a $4 \mathrm{~h}$ advance compared with the PDF-negative fifth LN and the DN1s (supplemental Fig. 1C, available at www.jneurosci.org as supplemental material).

Our results indicated that light entrainment of the DN2s requires PDF signaling from the LNs. We asked whether PDF is also required for their temperature entrainment. Under HC cycles, lack of PDF in the LNs did not prevent PER levels from oscillating in the DN2s (Fig. 4B). Similarly, PER oscillations in the DN2s were not altered by the selective ablation of the LNs through pdf-gal4-driven hid and reaper ( $r p r)$ expression (data not shown). We conclude that temperature entrainment of the larval DN2s does not depend on the PDF-expressing LNs, as opposed to their light entrainment.

In the absence of a clock in the DN2s, the larval LNs do not entrain to temperature cycles

Oscillation patterns in HC suggested that temperature entrainment of the larval LNs may depend on the DN2s. We first tried to 

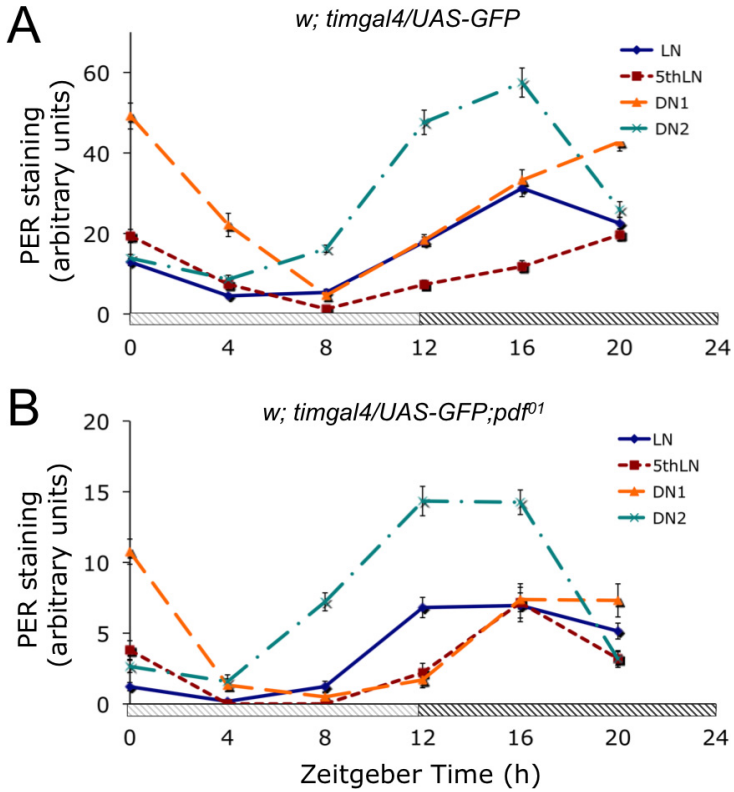

Figure 4. Time course of PER oscillations in the larval clock neurons in temperature cycles. Wild-type $(\boldsymbol{A})$ and $p d f^{71}$ mutant $(\boldsymbol{B})$ larvae were entrained in $\mathrm{HC}\left(25^{\circ} \mathrm{C} / 19^{\circ} \mathrm{C}\right)$ conditions from the second day of development. Brains were dissected every $4 \mathrm{~h}$ during an $\mathrm{HC}$ cycle (ZTO corresponds to the beginning of the hot period and ZT12 corresponds to its end). PER quantization was done as described in Figure 1 . The light-gray-striped and black-striped boxes on $x$-axes indicate hot and cold periods, respectively. Error bars represent the SEM for each neuronal group.

test this hypothesis by selectively ablating the DN2s but failed to find conditions killing them while preserving the LNs (data not shown). We thus used the selective restoration of PER expression in a per $^{\circ}$ background. Larvae with PER restored only in the PDFpositive LNs ( wper $^{0}$;pdf-gal4/+;UAS-per16/+), or only in the PDF-positive LNs and DN1s (wper ${ }^{\circ}$;cry-gal4-39/+;UAS-per16/

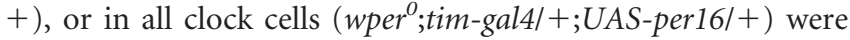
first tested in $\mathrm{LD}$ at $20^{\circ} \mathrm{C}$. PER oscillations were observed in the LNs of all three genotypes (supplemental Fig. 3, available at www. jneurosci.org as supplemental material). Such larvae were then entrained in $23-18^{\circ} \mathrm{C} \mathrm{HC}$ cycles and PER levels were measured the second day after release in constant conditions $\left(18^{\circ} \mathrm{C}\right)$ (see Materials and Methods). Temperature-entrained PER oscillations were seen in the LNs when PER expression was driven by tim-gal4, but not by either pdf-gal4 or cry-gal4-39 (Fig. 5; supplemental Fig. 4, available at www.jneurosci.org as supplemental material). Their phase was close to that of $\mathrm{per}^{+}$control brains. PER also oscillated with an evening phase in the DN2s of both the control larvae and the larvae with tim-gal4-driven PER expression (Fig. 5). Similar results were obtained for the TIM protein (data not shown). The rescue experiments thus show that the PDF-positive LNs entrain to thermocycles only if PER oscillations are present in other clock neurons, namely the DN2s and/or the PDF-negative fifth LN.

\section{Putative reciprocal connections between the larval LNs and DN2s}

The larval DN2s were reported to project to the contralateral hemisphere (Kaneko and Hall, 2000), but their precise targets, as well as their dendritic arborization, are unknown. We expressed the membrane CD8-GFP reporter under the control of tim-gal4 and cry-gal80 to restrict its expression among brain clock neurons to the DN2s (and fifth LN). On single confocal sections, a wide and dense dendritic-like arborization was seen to emerge from
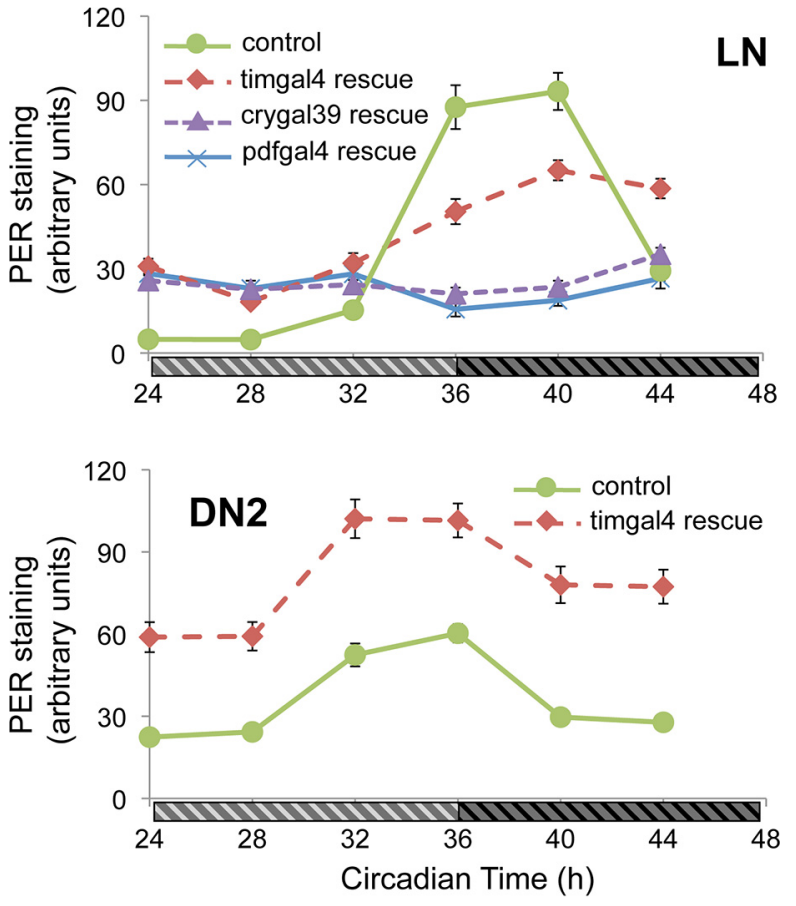

Figure 5. PER oscillations in the larval clock neurons in constant conditions after temperature entrainment. w;tim-gal4/UAS-gfp (control) larvae, as well as wper larvae with PER rescue only in the LNs (driven by pdf-gal4), or only in the LNs and DN1s (driven by cry-gal4-39), or in all clock cells (driven by tim-gal4), were entrained in $\mathrm{HC}\left(23^{\circ} \mathrm{C} / 18^{\circ} \mathrm{C}\right)$ conditions for at least $6 \mathrm{~d}$ from the second day of development, and then temperature was kept constant at $18^{\circ} \mathrm{C}$. Brains were dissected every $4 \mathrm{~h}$ during the second day in constant conditions (CT24 corresponds to the beginning of the subjective hot period and (T36 to its end). PER quantization was done as described in Figure 1. Error bars represent the SEM for each neuronal group (for the control DN2s, they are generally smaller than the symbols). Similar results were obtained at CT24CT28 and CT36 - CT40 for all genotypes in two additional experiments. ANOVA concluded that pdf-gal4- and cry-gal4-39-rescued PER variations in the LNs were not significantly different $(p>0.1)$ from each other, and that both were significantly different $(p<0.0001)$ from PER oscillations in the LNs of tim-gal4-rescued larvae. The light gray stripes and black stripes on medium gray background indicate subjective day and night, respectively.

the contralaterally heading DN2 tract, very close to the DN2 somas and following closely the curvature of the dorsal projections of the LNs (Fig. 6; supplemental Fig. 5, available at www. jneurosci.org as supplemental material). This large contact zone appears well suited to receive PDF signals from the LNs. Further down along the PDF-positive projections, at least three strongly GFP-labeled fibers could be followed (Fig. 6, arrowheads). One of them belongs to the fifth LN (data not shown). Continuity between the other two fibers and the wide arborization from the DN2s, more dorsally, could not be established unambiguously. However, based on their occurrence in the same confocal section, and on the absence of any other clock cell with strong GFP expression in this genotype, we propose that they indeed represent a hitherto unrecognized projection from the DN2s, possibly providing a reciprocal connection with the LNs.

The phase of adult activity rhythms after larval light or temperature entrainment is fixed by the phase acquired by the LNs, and not the DN2s

We asked whether the CRY-dependent inversion of PER oscillations in the larval DN2s would have consequences on circadian outputs. The only known larval output is the negative phototaxis rhythm described in foraging third-instar larvae (Mazzoni et al., 2005), but we were unable to observe it with our strains and 

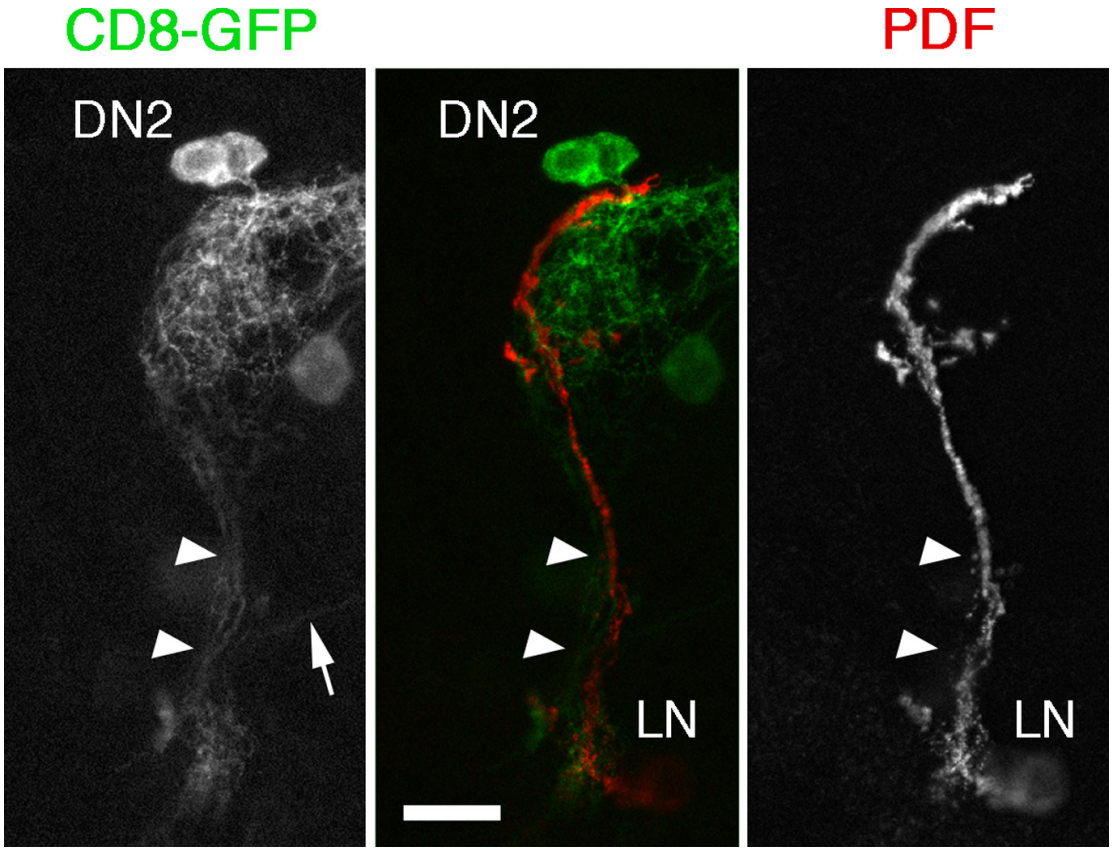

Figure 6. Anatomical evidence for connections between the LNs and the DN2s. Single confocal section from a w;tim-gal4/UASCD8-GFP; ;ry-gal80/ + larval brain, labeled with anti-PDF antibodies (right panel; red in middle panel). At least three GFP ${ }^{+}$fibers can be seen along the PDF-positive ones, most clearly between the arrowheads. The arrow points to fibers that presumably run in the posterior optic tract, coming from the I-LNv precursors already detectable before metamorphosis (Helfrich-Förster et al., 2007). A GFP ${ }^{+}$nonclock cell, which projects dorsally, is slightly out of focus. Note that, because of cry-gal80, there is no coexpression of GFP and PDF. Another sample is shown in supplemental Figure 5 (available at www.jneurosci.org as supplemental material). Scale bar, $20 \mu \mathrm{m}$.

A $y w ; p d f-G a l 4 / U A S-c r y$ yw;tim-Gal4/UAS-cry Adult light entrainment (LD)
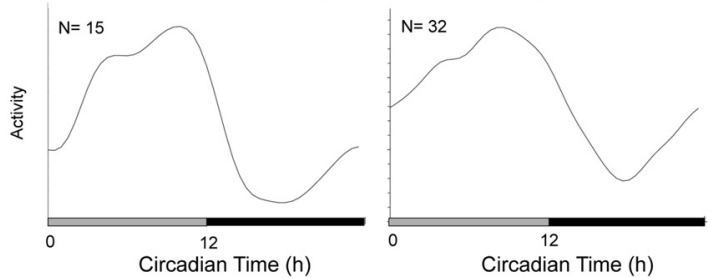

Larval light entrainment (LDD)
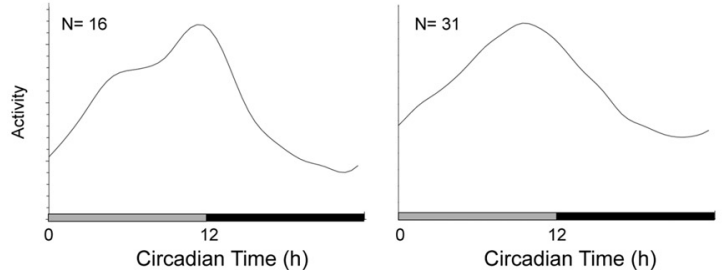

B Larval light entrainment (LDD)

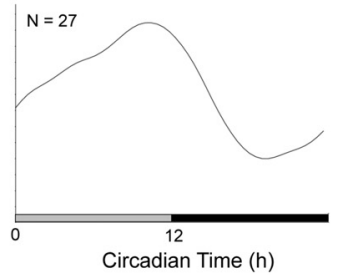

Larval $\mathrm{T}^{\circ} \mathrm{C}$ entrainment $(\mathrm{HCC})$

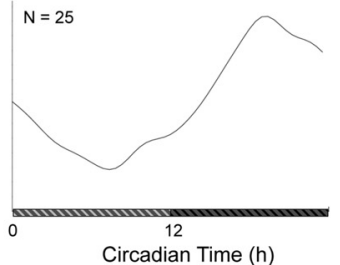

Figure 7. Phase of adult activity rhythms after larval entrainment by light or temperature. $A$, The activity profiles (waveforms) of adult flies with or without CRY-expressing DN2s were averaged over $6 \mathrm{~d}$ of DD and N flies, after larval (LDD) or adult (LD) entrainment by light. The light gray and black boxes indicate entrainment light and dark periods, respectively. Flies overexpressing CRY in all clock neurons (right) are compared with flies overexpressing CRY in PDF-positive LNs only (left). N, Number of flies. $\boldsymbol{B}$, Wild-type adult activity profiles were averaged over $6 \mathrm{~d}$ of DD and N flies, after larval entrainment by either $L D$ (top) or $H C$ (bottom) cycles. Both entrainment regimens were stopped before the first pupae appeared, and replaced by constant conditions (LDD: DD at $20^{\circ} \mathrm{C} ; \mathrm{HCC}: \mathrm{DD}$ at $\left.19^{\circ} \mathrm{C}\right) .0 \mathrm{n}$ the $x$-axis of the top panel, the light gray and black boxes indicate entrainment light and dark periods, respectively. 0 n the $x$-axis of the bottom panel, the boxes with light gray stripes or black stripes, on medium gray background, represent the entraining hot or cold periods, respectively.

experimental setup (data not shown). Since LD entrainment restricted to larval stages is sufficient to phase adult behavior (Sehgal et al., 1992; Kaneko et al., 2000; Malpel et al., 2004), we looked at adult activity rhythms in DD after larval-only or larval-to-adult entrainment. There was no obvious difference in phase or otherwise between flies with and without CRY in the DN2s, in either entrainment regimen (Fig. 7A).

We also measured the phase of locomotor activity rhythms in adults derived from larvae entrained in either HC or LD cycles, and then kept in constant conditions. After larval-only $\mathrm{HC}$ entrainment, peak activity occurred during the second one-half of the subjective night, as opposed to during the second one-half of the subjective day after larval-only LD entrainment (Fig. 7B; supplemental Fig. 6, available at www.jneurosci.org as supplemental material). Thus, entraining larvae by either temperature or light signals results in almost antiphased rhythms of adult activity, as predicted by the corresponding PER cycling patterns of the larval PDF-positive LNs (Figs. 1, 4, 5). These cells are indeed the only clock cells that function throughout metamorphosis (Kaneko et al., 1997; Helfrich-Förster et al., 2007).

\section{Discussion}

PDF and the light entrainment of CRYless clock neurons in the larval brain Although the absence of PDF severely affects Drosophila activity rhythms in DD (Renn et al., 1999), the exact function of the neuropeptide in the adult clock neuronal network remains unclear. In LD, PDF is required to produce a morning activity peak and to properly phase the evening peak, but not to entrain the brain clock (Renn et al., 1999; Grima et al., 2004). The behavioral phenotypes of PDF receptor mutants resemble that of the $p d f^{01}$ mutant (Hyun et al., 2005; Lear et al., 2005; Mertens et al., 2005). PDFR is expressed in all clock neurons except the large ventral lateral neurons (l-LNvs) (Shafer et al., 2008), supporting a role of PDF in maintaining phase coherence within the adult clock network in DD (Peng et al., 2003; Lin et al., 2004; Wu et al., 2008; Wulbeck et al., 2008). The loss of PER oscillations in the DN2s of $p d f^{01}$ larvae demonstrates a clear and novel role of PDF in transmitting not only phase information but also a synchronizing signal without which the receiving neurons are not entrained in LD.

Our results show that the CRY-less DN2s (Klarsfeld et al., 2004) are "blind" neurons that perceive light indirectly (Fig. 8 ). The PDF receptor rescue experiments strongly suggest that PDF acts on its receptor on the larval DN2s themselves, which are located in the vicinity of the LN axon terminals (Kaneko et al., 1997). Furthermore, we show here that the DN2s possess a wide and dense neuritic network that borders on the axons of the LNs over a large fraction of their length. 
However, we cannot rule out that expression of the receptor in the (PDF-negative) fifth $\mathrm{LN}$ is involved in synchronizing the DN2s downstream, through PDFindependent mechanisms.

The PDF-negative fifth LN is also a CRY-negative clock neuron (Klarsfeld et al., 2004), but it cycles in phase with the CRY-positive neurons of the larval brain (Kaneko et al., 1997; this study). The visual input to the PDF-expressing LNs appears sufficient to phase them normally even in $c r y^{b}$ mutants (Kaneko et al., 2000; Ivanchenko et al., 2001). It could thus be expected to entrain the CRY-less fifth LN in phase with the other larval LNs, as observed, in contrast to the CRY-less DN2s (Kaneko et al., 1997). A direct input from the visual system to the fifth $\mathrm{LN}$ is also consistent with its PDF-independent entrainment by LD cycles. Similarly, light entrainment of the larval DN1s in $c r y^{b}$ mutants (Kaneko et al., 2000) is consistent with their suggested connection to the visual system (Kaneko and Hall, 2000). Thus, the CRY-less DN2s would be the only larval clock neurons devoid of such a connection.

Adult eclosion rhythms depend on the PDF-expressing LNs (Blanchardon et al., 2001) and appear to require the PDFdependent clock that resides in the prothoracic gland (Myers et al., 2003). Since the larval DN2s project in the pars intercerebralis, a region of the brain that sends projections to the prothoracic gland (Siegmund and Korge, 2001), they could play a role in this physiologically important clock function. Our results raise the possibility that the damped PER oscillations in the DN2s of the $p d f^{01}$ mutants participate to their eclosion phenotype.

\section{A role of the larval DN2s in temperature entrainment}

The DN2s are the only larval clock neurons that are phased identically by light and temperature, but their temperature entrainment appears independent of any LN-derived signal. PER oscillations in the DN2s have a larger amplitude in $\mathrm{HC}$ cycles than in LD cycles, also suggesting a prominent role of temperature in their entrainment. Conversely, the molecular oscillations of the PDF-positive LNs have a larger amplitude in LD compared with $\mathrm{HC}$ cycles. In the latter, the molecular oscillations of the PDFexpressing LNs seem to follow those in the DN2s, with a large phase change compared with LD conditions. The DN1s and the PDF-negative fifth LN, in contrast, share another phase that is slightly advanced. Interestingly, behavioral (Yoshii et al., 2002; Busza et al., 2007) and transcriptome (Boothroyd et al., 2007) data in adult flies indicate that $\mathrm{HC}$ cycles result in a general phase advance relative to LD cycles. Cooperative synchronization of the clock by light and temperature likely requires temperature changes to act earlier than light changes since changes in temperature always lag behind changes in solar illumination in nature (Boothroyd et al., 2007). The very different relative phasing of the larval clock neurons in HC versus LD cycles suggests different ecological constraints on this life stage, spent mostly burrowed in

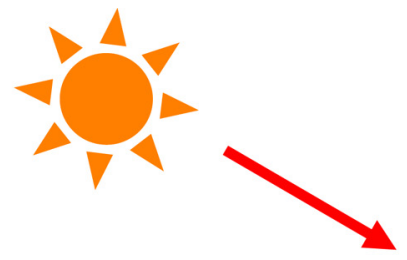

Temperature

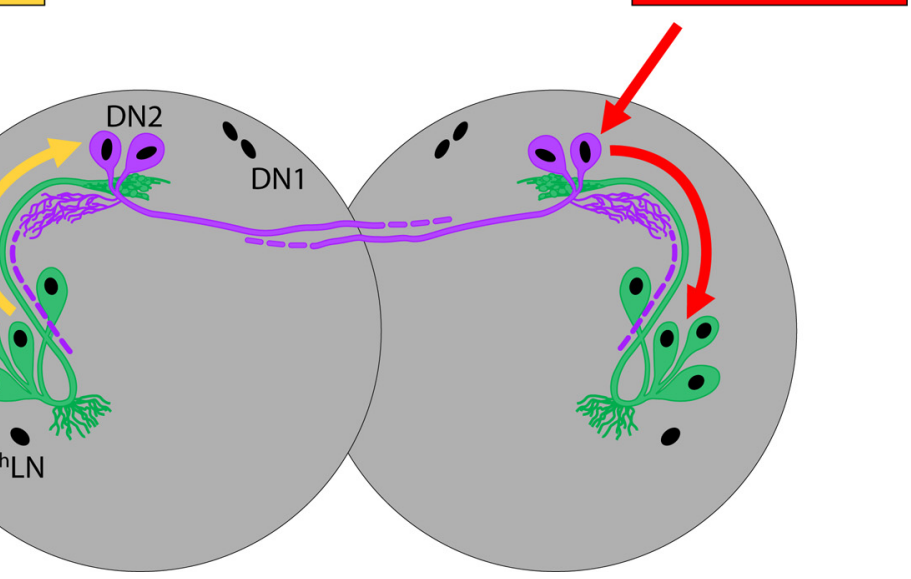

Figure 8. Model for larval clock responses to light and temperature cycles. In LD cycles, the larval DN2s are phased via PDF from the four PDF-positive LNs (in green), which are connected to the larval visual system and express the CRY protein. PDF-expressing LNs cycle less robustly than in LD, and with an almost opposite phase, corresponding to that of the DN2s (and differing from that of the other clock neurons). From the results of Figure 5, we propose that the LNs do not respond to $\mathrm{HC}$ cycles direct visual input), thus appear to form a functional subnetwork with the LNs, which are blind to temperature, but have both CRY expression and a direct visual input.

food, in which light may be a weaker Zeitgeber, and in which the lag between temperature and light changes may be quite different.

When a functional clock is absent from the DN2s (and the fifth LN), the larval PDF-expressing LNs are unable to entrain to thermocycles, whereas they autonomously entrain to LD cycles. It remains possible that autonomous temperature entrainment of the larval LNs (but not the DN2s) requires per transcriptional regulation, which the GAL4-UAS system is lacking. But our results demonstrate the existence of a control exerted on the LN clock by CRY-negative clock cells when temperature is the synchronizing cue. Although we cannot exclude a role of the fifth LN, the absence of autonomous photoperception by the DN2s nicely fits with a role in temperature entrainment. The high cycling amplitude of the DN2s in thermocycles and the locking of the phase of the LNs on that of the DN2s in these conditions strongly support their role in the temperature entrainment of the LNs.

Additional studies should investigate whether the DN2s communicate with the LNs via fibers that appear to run along the dorsal projection of the LNs. Alternatively, the dense dendriticlike network of the DN2s could ensure reciprocal exchanges between them and the LNs. We thus propose a model whereby, in the larval brain, the DN2s and the four PDF-positive LNs form a distinct subnetwork, with the LNs entraining the DN2s in LD, whereas the opposite is true in HC (Fig. 8). What becomes of their hierarchy in constant conditions, after entrainment stops? Their relative phases appear to change little at least during the first $2 \mathrm{~d}$ after entrainment, whether they have been set in antiphase by LD 
entrainment, or in phase by HC entrainment. This suggests that, whatever the entraining regimen, the LNs and the DN2s run autonomously in constant conditions. However, we cannot exclude that one of the two groups still dominates but requires more time after the end of entrainment to shift the phase of the other.

The rhythmic behavior of the adult flies that emerge from the temperature-entrained larvae is almost in antiphase compared with the one of flies entrained by light during the larval stage. This strongly suggests that the phase of the adult rhythms is set by the antiphasic oscillations of the larval PDF-positive LNs, consistent with these cells being the only neurons in which molecular cycling persists throughout metamorphosis (Kaneko et al., 1997; Helfrich-Förster et al., 2007). We thus believe that the large phase shift of adult activity can be accounted for simply by the large phase shift of molecular oscillations in the PDF-expressing LNs.

It is often assumed that temperature affects the molecular clock directly and identically in all clock cells, as opposed to light, which requires dedicated input pathways. However, in the adult, thermocycles phase the brain clock differently from all peripheral clocks, as judged from whole-tissue oscillations of a luciferase reporter enzyme (Glaser and Stanewsky, 2005). Recent data suggest that subsets of clock neurons in the Drosophila adult brain may indeed be dedicated to temperature entrainment. In experiments combining LD and HC entrainment, all DN groups, as well as the less studied lateral posterior neurons (LPNs), seem to preferentially follow thermocycles, whereas the other LNs preferentially follow LD cycles (Miyasako et al., 2007). Although adult $\mathrm{PDF}^{+}$LNs are able to entrain to thermocycles in the absence of any other functional clock, they do not seem to be required for (and actually slowed down) the temperature entrainment of activity rhythms, whereas the PDF-negative LPNs appear to play a prominent role in such conditions (Busza et al., 2007).

Our results indicate that a similar specialization toward light or temperature entrainment exists in the larval brain. The DN2s, which appear to be the most temperature-responsive clock neurons, are by themselves completely blind. Conversely, the four PDF-positive LNs, which may be the most light-sensitive clock neurons (with both CRY and the visual system as inputs), appear almost temperature blind, and depend on the DN2s for temperature entrainment. PER-negative DN2s do not allow PER oscillations in the larval LNs, suggesting that entrainment of the latter in HC cycles depends on clock function in the former. The hierarchy of clock neurons thus appears very different during entrainment of the clock network by one or the other Zeitgeber.

\section{References}

Bell-Pedersen D, Cassone VM, Earnest DJ, Golden SS, Hardin PE, Thomas TL, Zoran MJ (2005) Circadian rhythms from multiple oscillators: lessons from diverse organisms. Nat Rev Genet 6:544-556.

Blanchardon E, Grima B, Klarsfeld A, Chélot E, Hardin PE, Préat T, Rouyer F (2001) Defining the role of Drosophila lateral neurons in the control of circadian activity and eclosion rhythms by targeted genetic ablation and PERIOD protein overexpression. Eur J Neurosci 13:871-888.

Boothroyd CE, Wijnen H, Naef F, Saez L, Young MW (2007) Integration of light and temperature in the regulation of circadian gene expression in Drosophila. PLoS Genet 3:e54.

Busza A, Murad A, Emery P (2007) Interactions between circadian neurons control temperature synchronization of Drosophila behavior. J Neurosci 27:10722-10733.

Chang DC (2006) Neural circuits underlying circadian behavior in Drosophila melanogaster. Behav Processes 71:211-225.

Dunlap JC, Loros JJ, DeCoursey PJ, eds (2004) Chronobiology. Biological timekeeping. Sunderland, MA: Sinauer Associates.

Emery P, Stanewsky R, Helfrich-Förster C, Emery-Le M, Hall JC, Rosbash M
(2000) Drosophila CRY is a deep brain circadian photoreceptor. Neuron 26:493-504.

Glaser FT, Stanewsky R (2005) Temperature synchronization of the Drosophila circadian clock. Curr Biol 15:1352-1363.

Grima B, Lamouroux A, Chélot E, Papin C, Limbourg-Bouchon B, Rouyer F (2002) The F-box protein SLIMB controls the levels of clock proteins PERIOD and TIMELESS. Nature 429:178-182.

Grima B, Chélot E, Xia R, Rouyer F (2004) Morning and evening peaks of activity rely on different clock neurons of the Drosophila brain. Nature 431:869-873

Hall JC (2003) Genetics and molecular biology of rhythms in Drosophila and other insects. Adv Genet 48:1-280.

Hamblen M, Zehring WA, Kyriacou CP, Reddy P, Yu Q, Wheeler DA, Zwiebel LJ, Konopka RJ, Rosbash M, Hall JC (1986) Germ-line transformation involving DNA from the period locus in Drosophila melanogaster: overlapping genomic fragments that restore circadian and ultradian rhythmicity to per0 and per- mutants. J Neurogenet 3:249-291.

Helfrich-Förster C, Winter C, Hofbauer A, Hall JC, Stanewsky R (2001) The circadian clock of fruit flies is blind after elimination of all known photoreceptors. Neuron 30:249-261.

Helfrich-Förster C, Edwards T, Yasuyama K, Wisotzki B, Schneuwly S, Stanewsky R, Meinertzhagen IA, Hofbauer A (2002) The extraretinal eyelet of Drosophila: development, ultrastructure and putative circadian function. J Neurosci 22:9255-9266.

Helfrich-Förster C, Shafer OT, Wülbeck C, Grieshaber E, Rieger D, Taghert P (2007) Development and morphology of the clock-gene-expressing lateral neurons of Drosophila melanogaster. J Comp Neurol 500:47-70.

Hyun S, Lee Y, Hong ST, Bang S, Paik D, Kang J, Shin J, Lee J, Jeon K, Hwang S, Bae E, Kim J (2005) Drosophila GPCR Han is a receptor for the circadian clock neuropeptide PDF. Neuron 48:267-278.

Ivanchenko M, Stanewsky R, Giebultowicz JM (2001) Circadian photoreception in Drosophila: functions of cryptochrome in peripheral and central clocks. J Biol Rhythms 16:205-215.

Kaneko M (1998) Neural substrates of Drosophila rhythms revealed by mutants and molecular manipulations. Curr Opin Neurobiol 8:652-658.

Kaneko M, Hall JC (2000) Neuroanatomy of cells expressing clock genes in Drosophila: transgenic manipulation of the period and timeless genes to mark the perikarya of circadian pacemaker neurons and their projections. J Comp Neurol 422:66-94.

Kaneko M, Helfrich-Förster C, Hall JC (1997) Spatial and temporal expression of the period and timeless genes in the developing nervous system of Drosophila: newly identified pacemaker candidates and novel features of clock gene product cycling. J Neurosci 17:6745-6760.

Kaneko M, Hamblen MJ, Hall JC (2000) Involvement of the period gene in developmental time-memory: effect of the perShort mutation on phase shifts induced by light pulses delivered to Drosophila larvae. J Biol Rhythms 15:13-30.

Kaushik R, Nawathean P, Busza A, Murad A, Emery P, Rosbash M (2007) PER-TIM interactions with the photoreceptor Cryptochrome mediate circadian temperature responses in Drosophila. PLoS Biol 5:e146.

Klarsfeld A, Leloup JC, Rouyer F (2003) Circadian rhythms of locomotor activity in Drosophila. Behav Processes 64:161-175.

Klarsfeld A, Malpel S, Michard-Vanhée C, Picot M, Chélot E, Rouyer F (2004) Novel features of cryptochrome-mediated photoreception in the brain circadian clock of Drosophila. J Neurosci 24:1468-1477.

Konopka RJ, Pittendrigh C, Orr D (1989) Reciprocal behaviour associated with altered homeostasis and photosensitivity of Drosophila clock mutants. J Neurogenet 6:1-10.

Lear BC, Merrill CE, Lin JM, Schroeder A, Zhang L, Allada R (2005) A G protein-coupled receptor, groom-of-PDF, is required for PDF neuron action in circadian behavior. Neuron 48:221-227.

Lin Y, Stormo GD, Taghert PH (2004) The neuropeptide PDF coordinates pacemaker interactions in the Drosophila circadian system. J Neurosci 24:7951-7957.

Malpel S, Klarsfeld A, Rouyer F (2002) Larval optic nerve and adult extraretinal photoreceptors sequentially associate with the clock neurons during Drosophila brain development. Development 129:1443-1453.

Malpel S, Klarsfeld A, Rouyer F (2004) Circadian synchronization and rhythmicity in larval photoperception-defective mutants of Drosophila. J Biol Rhythms 19:10-21.

Matsumoto A, Matsumoto N, Harui Y, Sakamoto M, Tomioka K (1998) Light and temperature cooperate to regulate the circadian locomotor 
rhythm of wild type and period mutants of Drosophila melanogaster. J Insect Physiol 44:587-596.

Mazzoni EO, Desplan C, Blau J (2005) Circadian pacemaker neurons transmit and modulate visual information to control a rapid behavioral response. Neuron 45:293-300.

Mertens I, Vandingenen A, Johnson EC, Shafer OT, Li W, Trigg JS, De Loof A, Schoofs L, Taghert PH (2005) PDF receptor signaling in Drosophila contributes to both circadian and geotactic behaviors. Neuron 48:213-219.

Miyasako Y, Umezaki Y, Tomioka K (2007) Separate sets of cerebral clock neurons are responsible for light and temperature entrainment of Drosophila circadian locomotor rhythms. J Biol Rhythms 22:115-126.

Myers EM, Yu J, Sehgal A (2003) Circadian control of eclosion: interaction between a central and peripheral clock in Drosophila melanogaster. Curr Biol 13:526-533.

Park JH, Helfrich-Förster C, Lee G, Liu L, Rosbash M, Hall JC (2000) Differential regulation of circadian pacemaker output by separate clock genes in Drosophila. Proc Natl Acad Sci U S A 97:3608-3613.

Peng Y, Stoleru D, Levine JD, Hall JC, Rosbash M (2003) Drosophila freerunning rhythms require intercellular communication. PLoS Biol 1:E13.

Renn SC, Park JH, Rosbash M, Hall JC, Taghert PH (1999) A pdf neuropeptide gene mutation and ablation of PDF neurons each cause severe abnormalities of behavioral circadian rhythms in Drosophila. Cell 99:791-802.

Rensing L, Ruoff P (2002) Temperature effect on entrainment, phase shifting, and amplitude of circadian clocks and its molecular bases. Chronobiol Int 19:807-864.

Rieger D, Stanewsky R, Helfrich-Förster C (2003) Cryptochrome, compound eyes, $\mathrm{H}-\mathrm{B}$ eyelets and ocelli play different roles in the entrainment and masking pathway of the locomotor activity rhythm in the fruit fly Drosophila melanogaster. J Biol Rhythms 18:377-391.

Roenneberg T, Daan S, Merrow M (2003) The art of entrainment. J Biol Rhythms 18:183-194.

Sehgal A, Price J, Young MW (1992) Ontogeny of a biological clock in Drosophila melanogaster. Proc Natl Acad Sci U S A 89:1423-1427.

Shafer OT, Helfrich-Förster C, Renn SC, Taghert PH (2006) Reevaluation of Drosophila melanogaster's neuronal circadian pacemakers reveals new neuronal classes. J Comp Neurol 498:180-193.

Shafer OT, Kim DJ, Dunbar-Yaffe R, Nikolaev VO, Lohse MJ, Taghert PH (2008) Widespread receptivity to neuropeptide PDF throughout the neuronal circadian clock network of Drosophila revealed by real-time cyclic AMP imaging. Neuron 58:223-237.

Sidote D, Majercak J, Parikh V, Edery I (1998) Differential effects of light and heat on the Drosophila circadian clock proteins PER and TIM. Mol Cell Biol 18:2004-2013.

Siegmund T, Korge G (2001) Innervation of the ring gland of Drosophila melanogaster. J Comp Neurol 431:481-491.

Stanewsky R, Frisch B, Brandes C, Hamblen-Coyle MJ, Rosbash M, Hall JC (1997) Temporal and spatial expression patterns of transgenes containing increasing amounts of the Drosophila clock gene period and a lac $Z$ reporter: mapping elements of the PER protein involved in circadian cycling. J Neurosci 17:676-696.

Stoleru D, Peng Y, Agosto J, Rosbash M (2004) Coupled oscillators control morning and evening locomotor behavior of Drosophila. Nature 431:862-868.

Veleri S, Rieger D, Helfrich-Förster C, Stanewsky R (2007) HofbauerBuchner eyelet affects circadian photosensitivity and coordinates TIM and PER expression in Drosophila clock neurons. J Biol Rhythms 22:29-42.

Wheeler DA, Hamblen-Coyle MJ, Dushay MS, Hall JC (1993) Behavior in light-dark cycles of Drosophila mutants that are arrhythmic, blind, or both. J Biol Rhythms 8:67-94.

Wulbeck C, Grieshaber E, Helfrich-Forster C (2008) Pigment-dispersing factor (PDF) has different effects on Drosophila's circadian clocks in the accessory medulla and in the dorsal brain. J Biol Rhythms 23:409-424.

Wu Y, Cao G, Nitabach MN (2008) Electrical silencing of PDF neurons advances the phase of non-PDF clock neurons in Drosophila. J Biol Rhythms 23:117-128.

Yoshii T, Sakamoto M, Tomioka K (2002) A temperature-dependent timing mechanism is involved in the circadian system that drives locomotor rhythms in the fruit fly Drosophila melanogaster. Zoolog Sci 19:841-850.

Yoshii T, Heshiki Y, Ibuki-Ishibashi T, Matsumoto A, Tanimura T, Tomioka K (2005) Temperature cycles drive Drosophila circadian oscillation in constant light that otherwise induces behavioural arrhythmicity. Eur J Neurosci 22:1176-1184.

Yoshii T, Fujii K, Tomioka K (2007) Induction of Drosophila behavioral and molecular circadian rhythms by temperature steps in constant light. J Biol Rhythms 22:103-114.

Zeng H, Hardin PE, Rosbash M (1994) Constitutive overexpression of the Drosophila period protein inhibits period mRNA cycling. EMBO J 13:3590-3598.

Zhou L, Schnitzler A, Agapite J, Schwartz LM, Steller H, Nambu JR (1997) Cooperative functions of the reaper and head involution defective genes in the programmed cell death of Drosophila central nervous system midline cells. Proc Natl Acad Sci U S A 94:5131-5136. 